ACTA UIVERSITATIS CIBINIENSIS - TECHNICAL SERIES

Vol. LXV 2014

\title{
BROADENING THE CONCEPT OF POKA YOKE BEYOND AUTOMOTIVE INDUSTRY
}

\author{
ALEX MӐGDOIU \\ Faculty of Engineering, "Lucian Blaga" University, Sibiu, Romania, magdoiu@yahoo.com \\ CONSTANTIN OPREAN \\ Faculty of Engineering, "Lucian Blaga" University, Sibiu, Romania, presedinte@ulbsibiu.ro
}

\begin{abstract}
This paper will present the development of the poka yoke systems, which were initially created and implemented just for the automotive industry, also to other domains. For some fields like medicine, constructions or software development there are already articles available on the benefits brought by poka yoke systems' implementation. Besides these domains the article will contribute to the recognition of poka yoke systems in fields where, although error proofing systems are used, no link to the poka yoke method was made by the authors, creators and users.
\end{abstract}

Key words: Poka yoke, mistake proofing, error prevention.

\section{Introduction}

Poka yoke is a Japanese method of quality assurance, developed in the 60s by Shingo Shigeo, one of the industrial engineers that contributed to the creation of the best known total quality management system, TPS (Toyota Production System). Therefore the method appeared and was developed in the automotive industry. The translation from Japanese of the two words naming this method, "poka" - error and "yoke" - to avoid, gives an idea of its scope.

The poke yoke method has as a starting point the idea that the influence of the operator in an automated manufacturing process has not been previously sufficiently analysed. The worker cannot be treated similar to a machine, as his state of mind influences significantly the effectiveness and power of concentration leaving open at any time the possibility of a mistake such as: forgetting some steps of the process, assembling the wrong components or even the complete omission of a component assembly, misunderstanding or misinterpretation of a processes and many others. With the help of poka yoke systems it is intended to avoid such errors in the manufacturing process (Binner, 1996).

As a preventive element poka yoke is a simple mechanism or device, such as a frame or support basing itself for example on asymmetrical design which helps to ensure that the human element is no longer allowed to adversely affect the manufacturing process because any errors caused by his emotional state are detected (Schmitt, 2010).

\section{Poka yoke studies for other industries}

XXI century has brought with it increased interest for the poka yoke concept and thus adaptation to certain areas that have no connection to the auto industry or the Toyota Production System. This creates works that recognize the importance of the concept developed by Shingo Shigeo and the benefits of implementing such systems in constructions (Ramin, 2011; Dos Santos, 1999) and in software development (Robinson, 1997; Nataraj, 2013).

But the most common new area in the new articles on poka yoke is the field of medicine. Here, as in the automotive or aviation industry, the impact of human error can be critical as it can lead to loss of lives.

Hinckley (2007) deals with preventing errors using the classic point of view for poka yoke systems developed by Shingo Shigeo, namely various mechanisms that hinder the attainment of noncompliant products from different manufacturing or laboratory analyses because of inconsistent handling 
by operators. Whether it is automated or semi-automated lines of production for drugs or various machines and laboratory equipment, the author proposes various methods and techniques for avoiding errors, without naming them poka yoke, with which the human factor is controlled or advised of mistakes.

Saint Maurice et. al. (2011) and Carboneau (2000) propose a broader concept than the poke yoke developed by the creator of this quality improvement technique. Thus Saint Maurice et. al. propose a field widening for the poka yoke technique by using them to avoid human errors in activities not directly related to a manufacturing process. The authors present different applications of the poka yoke concept for medical processes in hospitals such as those related to anesthesia. Examples like color-coded labeling of syringes based on the anesthetic type or of clear and visible patients bed tilt at $30^{\circ}$ to prevent pneumopathy for patients who are intubated in intensive care

Carboneau focuses on changing the concept of human error in the medical field for drug overdose from the current focused detection to a prevention concept. The author also presents worrying figures from the healthcare system on the number of deaths due to medication errors. He estimates the error rate in prescribing appropriate medication and dosage between 4 and 6\% in the United States. As an example of poka yoke Carboneau presents a solution implemented by a drug manufacturer that changed the packaging of dangerous medication from packs with several doses in a single dose packaging, so that both the pharmacist and the patient cannot make an error that causes serious health problems or even death.

The most comprehensive article regarding error prevention in the medical field is carried out by Grout (2007). Not only that he proposes Shingo Shigeo's concept and other quality techniques to improve the quality practices in medicine, but he also extends these ideas with multiple examples of mechanisms that avoid errors for various medical processes in hospitals.

Broadening the concept of poka yoke in its application and adaptation to the medicine field was done by various authors starting from the ideas of this method's creator. They named these mechanisms and techniques if not poka yoke -as Shingo Shigeo did- methods for human error avoidance.

\section{Broadening poka yoke}

This chapter will present a further broadening of the initial poka yoke concept to different fields and in other forms, without being seen or recognized by the authors, creators or users as part of the method developed by Shingo Shigeo.

\subsection{Management}

The concept of poka yoke can be expanded easily to project management where, without being designated as poka yoke systems, different software solutions help project managers (PMs) to perform better by avoiding planning errors.

One of the most important responsibilities of a project manager is to allocate resources in the time schedule of the project. Generally the same resources (people and/or equipment) are accessed by more managers in the implementation of their projects. Thus, a common error is a double planning of the same resource in the same time slot by different PMs or even by the same one but in two different projects. By using Microsoft Project Professional it is possible to define global resources, that every manager will be able to access and reserve. In case of a resource planning over its defined capacity for a specific period of time, the software is automatically alerting the respective project managers. Managers have therefore the opportunity to reschedule their activities so that available resources do not affect the time schedule of the projects. By avoiding the possible planning errors described above, the presented software has the function of a poka yoke system for error prevention.

The same program presented above or similar ones can be considered a poka yoke system even when talking only about the timing planning of a project. Besides the activities on the critical path, within a complex project there are many other activities which have smaller planning restrictions. However, quite often these activities are neglected for a long period of time, until they actually delay the realization of the whole project. If a project planning software is used such situations will be avoided. The program alerts the PM when an activity is likely to delay the project schedule so that he can react and prevent this by focusing on the neglected activity.

Equally important to the success of a project is that its budget is properly monitored and allocated to the necessary activities. Also for these operations there are many applications that help the project manager and due to the possibility of these software solutions to prevent the errors of the PM, they can be defined as poka yoke systems.

Microsoft Project allows the user to define for each resource a fixed usage cost or a variable cost depending on the duration of use. This permits to have a total cost estimation of the project from the 
planning phase but also highlights costs till a time point in the project implementation. Available budget overrun can thus be identified in advance allowing the project manager to take actions to prevent project failure.

Changes have become nowadays one of the biggest challenges of the managers. The difficulty is determined by the complexity of the changes which increased mainly due to the rapid technological advance and the high rate of the society's development. Therefore, in order to assist managers, software applications have been developed which prevent them from taking the wrong decisions. By an accurate modelling of the system, it is possible to assess the impact of various change scenarios. The wrong decision is prevented by interpreting the results obtained from the simulations.

ChangeXpert (Nae, 2013) is an online application that allows consultants and managers to assess the impact and benefits of changes. By comparing different implementation scenarios, the application supports its users in making better decisions. The analysis of different scenarios is based on detailed reports, which are automatically generated, containing information about the proposed investment profitability. ChangeXpert is an easy to use program that automates complex calculations and gives credibility by allowing the figures verification. This verification is done by viewing interactive diagrams and also by providing a dynamic analysis for the changes' impact.

\subsection{Logistics}

The Kanban system, for production planning and for the control of the supply chain, as it was proposed by Taiichi Ohno is based on cards that contain basic information of this system such as material and quantity. Both the system and the cards have developed over time and the organizations that have implemented the Kanban system have tried to adapt it to their needs and production model. In order to avoid any mistakes as for example in the warehouse when the material number is incorrectly read by the operator or the material delivery at another place than the one requested on the card, different poka yoke systems were introduced for the prevention of errors.

By implementing a bar code on the Kanban card, which contains the material number, the possibility of incorrectly identifying the material was eliminated. The following sequence of work, in addition to shortening the time and increasing the efficiency of the raw material supply on the line, acts also as a poka yoke system that ensures the delivery of the right material in the right amount at the correct time and the right place:

- When a box of material was emptied, its Kanban card is placed in the appropriate place on the line;

- The responsible logistic operator comes at predefined times, on each line and scans the bar code representing the location where he is and then the bar codes of the Kanban cards found;

- Automatically in the warehouse a material release sheet is printed. This document contains data such as material number, quantity, storage location in the warehouse, place where the material has to be supplied and the predefined route that will be used for bringing the material on the line;

- After predefined time intervals all the release sheets for a route are taken and the needed materials are collected;

- Material collection is done by scanning the release sheet, the warehouse location from which the material is collected and the material box;

- Once all the materials are collected, the logistic operator transports them to the production lines, where he has to scan the location where the material is supplied and the box that he brought there.

By using this sequence, errors that can normally appear in the operations are detected and thus a logistic poka yoke is realized. Figure 1 presents part of the sequence that was introduced above. 
Figure 1: Poka yoke Kanban system operation

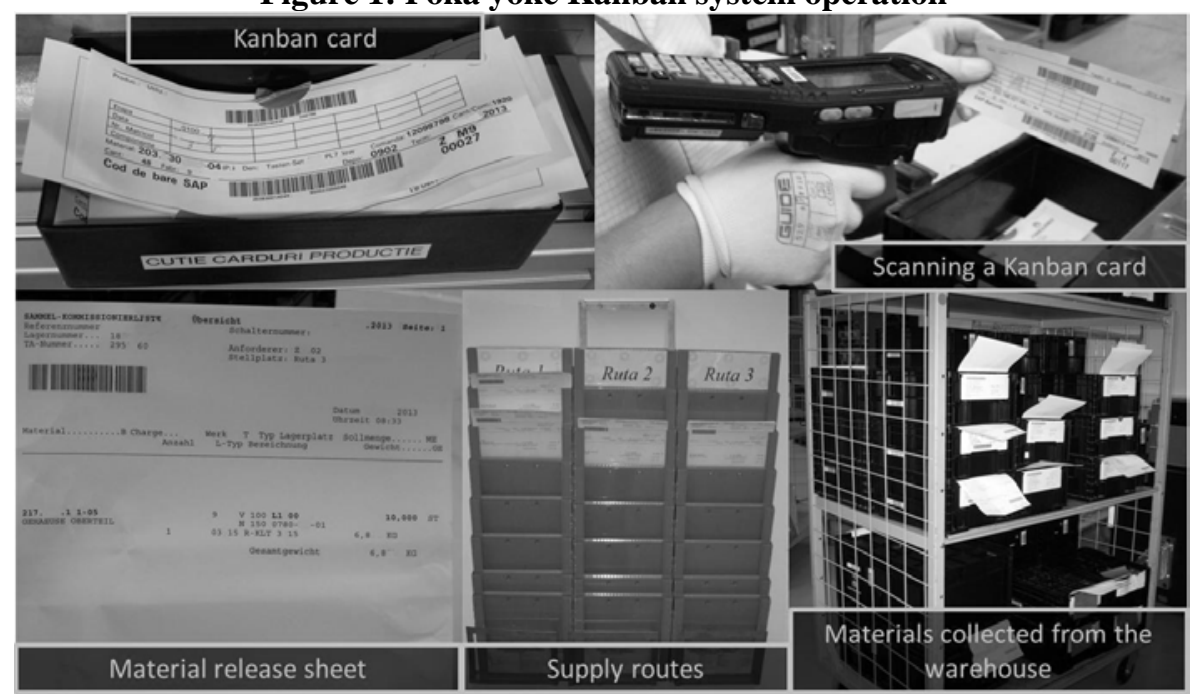

\subsection{Daily activities}

The poka yoke systems are meanwhile present in our life and in our daily activities. Sometimes we do not realize that their development and implementation reached so widely. Starting from the mechanical devices designed in order to correct the errors occurring on the production lines in the automotive industry, today we are surrounded by a multitude of error protection systems; although they are not designated as poka yoke systems.

The spell checking and correction functions, which are offered by most of the text editing programs and recently also by the email programs are some examples of anti-error systems; without being called poka yoke systems they fulfill all their characteristics. The system does not offer a guaranteed protection, but it has reached a very high level, especially for checking the English texts. The direction of development is very clear, so we can expect in the coming years an increasing quality of the checking.

Another example of a very simple poka yoke system that has helped to raise the quality of the office work is the warning that appears when trying to send an email without a subject. The messages that do not contain a subject may not pass some automatic filters and thus may not get to the recipient. Even if they reach the destination, these messages risk to be ignored by the people who receive daily a large number of emails and an ulterior search can be difficult for a message that doesn't have a suggestive title. In figure 2 you can see how the user is informed of the lack of the subject at the email he is trying to send.

Figure 2: Alert for sending an email without a subject

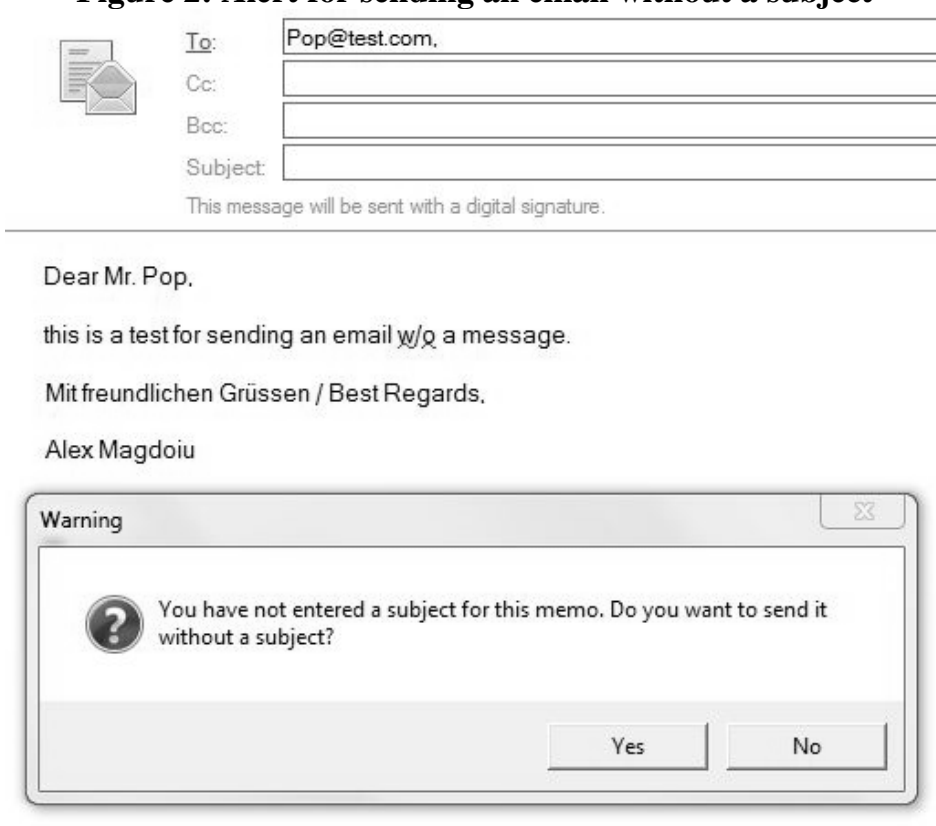


Leaving the software field, examples of poka yoke systems are found in many products used every day. Beginning with electrical or multimedia connectors that due to their asymmetry do not allow a wrong connection, to the kitchen where the microwave oven works only with the door closed or the hotplate that indicates the high temperature even after shutting it down.

The car comes also with a number of poka yoke systems, some of them developing in time from one form of poka yoke system to another, for an easy and comfortable use. For instance, in order to prevent the forgetfulness of the lights on, most of the manufacturers have initially implemented a warning poka yoke system that alerts the driver by means of a sounding alarm when leaving the car without switching off the lights. In time this system has evolved to a control poka yoke system that turns off automatically the lights when leaving the car.

A similar evolution happened at the fuel tank cap, where in order to avoid forgetting the cap in the gas station, different warning and control poka yoke systems have been tried. Most of them were based on hanging the cap with a short wire or locking the car keys in the opened cap. Currently most of the manufacturers chose the solution of the complete removal of the cap and its replacing with a flap that eliminates completely the risk of driving with the open tank, while increasing the comfort in utilization.

A very important system, preventing in time many traffic accidents is represented by the vibrating bands along the roadsides or the horizontal ones preceding the dangerous curves. Although not known as poka yoke systems, their role to avoid possible driving errors allows their classification in the concept developed by Shingo Shigeo.

\subsection{Services}

Poka yoke has also been introduced in the service industries in order to eliminate mistakes and their effect on customers. A good example of a poka yoke system in services is provided by the usage of counter tickets (take-a-number system). Installing such a system ensures that the correct order in which customers must be served is kept. Another example is the introduction of electronic payment systems that eliminate the risk of charging the customer with the wrong amount of money. The use of this system eliminates the inconvenience of not being able to provide change due to the lack of the right amount from the register.

Other examples from this category would be to use different colors for the service staff uniforms depending on their specialty. When cruising, the uniforms of the bar waiters, restaurant waiters, entertainment team members, casino employees, receptionists and cleaning staff are different so guests know to which crew member they have to address in order to satisfy their desires.

One of the most important contributions in this field was the introduction of the barcode in the distribution companies. By implementing such a poka yoke system, the work quality of these companies substantially increased, by avoiding data entry errors by the operators of the package collection and distribution centers whose error rate was 10 mistakes per 1000 characters introduced in the information system (Supply Chain Services, 2013).

\section{Conclusions}

The poka yoke method is part of the Toyota Production System and therefore it appeared and was developed by its creator, Shingo Shigeo for the automotive industry. The paper analyzed existing contemporary industries but also various fields and everyday life, where numerous examples of poka yoke systems were identified although they have not been treated as such in the literature. The most significant and relevant examples were classified according to the area in which they can be applied, namely management, logistics, daily activities and services.

Basically the work analysed only the tip of the iceberg in terms of broadening the application field of poka yoke systems, presenting only a part of the many examples, technologies and methods that are based on the theory founded by Shingo Shigeo, without being labelled as such. Poka yoke applications and systems are present around us in every area we work and operate and even if not perceived, they certainly help us be more productive and prevent us from doing unintentional errors that might affect the output quality.

\section{Acknowledgements}

This work was supported by the Ministry of Education and Research through POSDRU/ CPP107/DMI1.5/S/76851 project. 


\section{References}

- Binner, H.F. (1996) Umfassende Unternehmensqualität. Berlin Heidelberg: Springer.

- Carboneau, C. (2000) Reduce Medication Errors By Mistake Proofing, Quality Progress, vol. 33, no. 8, p. $8-10$.

- Grout, J. (2007) Mistake-Proofing the Design of Health Care Processes. Rockville: AHRQ Publication.

- Hinckley, C. M. (2007) Combining mistake-proofing and Jidoka to achieve world class quality in clinical chemistry, Accred. Quality Assurance, vol. 12, p. 223 - 230.

- Nae, L.; Balan, E.; Constantin, G.; Bendic V. (2013) Forecast the impact of change in a manufacturing environment, WSEAS International Conference on Industrial and Manufacturing Technologies (INMAT13), p. 202 - 205.

- Nataraj, K. (2013) How to Use Poka-Yoke (Mistake Proofing) Technique to Improve Software Quality. Available at http://www.softwaretestinghelp.com/poka-yoke/.

- Ramin, S.; et. al. (2011) Improving Productivity through Mistake-proofing of Construction Processes, CSIT International Conference on Intelligent Building and Management, vol. 5, p. 280 - 284, Singapore.

- Robinson, H. (1997) Using Poka-Yoke Techniques for Early Defect Detection, Sixth International Conference on Software Testing Analysis and Review, p. $134-145$.

- de Saint Maurice, G.; et. al. (2011) Comprendre la notion de détrompage, Annales Françaises d'Anesthésie et de Réanimation, no. 30, p. 51 - 56.

- Dos Santos, A.; Powell, J. (1999) Potential of Poka-Yoke Devices to Reduce Variability in Construction, Proceedings IGLC-7 26-28 July 1999, University of California, Berkeley, CA, USA, p. $51-62$.

- Schmitt, R.; Pfeifer, T. (2010) Qualitätsmanagement Strategien-Methoden-Techniken. München: Carl Hanser.

- Supply Chain Services (2013) Advantages Abound with Barcode Labelers. Available at http://supplychainservices.com/news/blog/2013/8/15/advantages-abound-with-barcode-labelers/. 